\title{
Low-temperature properties of monoalcohol glasses and crystals
}

\author{
M.A. Ramos, M. Hassaine, and B. Kabtoul \\ Laboratorio de Bajas Temperaturas, Departamento de Física de la Materia Condensada, and Instituto de Ciencia \\ de Materiales "Nicolás Cabrera", Universidad Autónoma de Madrid, Cantoblanco, Madrid E-28049, Spain \\ E-mail: miguel.ramos@uam.es \\ R.J. Jiménez-Riobóo \\ Instituto de Ciencia de Materiales de Madrid (ICMM-CSIC), Cantoblanco, Madrid E-28049, Spain
}

I.M. Shmyt'ko

Institute of Solid State Physics of Russian Academy of Sciences, Chernogolovka 142432, Moscow dist., Russia

\author{
A.I. Krivchikov, I.V. Sharapova, and O.A. Korolyuk \\ B. Verkin Institute for Low Temperature Physics and Engineering of the National Academy of Sciences of Ukraine \\ 47 Lenin Ave., Kharkov 61103, Ukraine
}

Received September 24, 2012

\begin{abstract}
We review and jointly discuss both earlier and recent experiments conducted by us on simple aliphatic glassforming monoalcohols at low temperatures, including specific heat, thermal conductivity, Brillouin scattering and x-ray diffraction experiments. The family of simple monoalcohols constitutes an interesting model system to explore different relevant issues concerning molecular glass-forming liquids, low-temperature universal properties of glasses, and even the glass transition phenomenon itself. More specifically, we discuss the role played by the molecular aspect ratio in vitrification/crystallization kinetics, the reported appearance of particular cases of polymorphism (in ethanol) and polyamorphism (in butanol), and especially the influence of position isomerism and the location of the hydrogen bond on the lattice dynamics and hence on the low-temperature universal properties of glasses.
\end{abstract}

PACS: 65.60.+a Thermal properties of amorphous solids and glasses: heat capacity, thermal expansion, etc.;

65.40.Ba Heat capacity;

66.70.Hk Glasses and polymers;

63.50.-x Vibrational states in disordered systems.

Keywords: glass transition, monohydroxy alcohols, low-temperature thermal properties of glasses, specific heat, polymorphism, molecular glasses.

\section{Introduction}

The very nature of glasses (and of disordered condensed matter, in general), as well as glass-transition related phenomena, still represents an open challenge in condensed-matter physics [1] and chemical physics. Also, the universal properties (thermal, vibrational, acoustic, dielectric, etc.) exhibited by glasses or amorphous solids at low temperatures or low frequencies [2] continue to be a major controversial issue.
On the other hand, much interest has been recently paid to molecular glass-forming liquids. One practical reason for such an interest is that these liquids usually solidify either into crystal or glassy states at temperatures between liquid-nitrogen and room temperatures, therefore providing easy access to the different states of the substance for many experimental techniques. They often also include manifestations of polymorphism and even polyamorphism [3] (i.e., the apparent existence of first-order transitions between two liquid states of a single-component substance 
or, more generally, between two distinct amorphous states of that substance), that makes them even more interesting to be studied.

In this paper, we briefly review and discuss the main calorimetric and thermodynamic results found in the family of aliphatic monohydroxy alcohols-or monoalcohols$\mathrm{H}\left(\mathrm{CH}_{2}\right)_{n} \mathrm{OH}$ for $n=1,2,3,4$, both from our own experiments and from the literature. First, we will discuss how the gradual elongation of the molecule with increasing number of ethyl groups affects the vitrification vs crystallization kinetics for primary monoalcohols (that is, when the hydrogen bond is fixed at the end of the molecule). We will also address briefly the reported presence of crystalline polymorphism in ethanol and polyamorphism in butanol. Then, we will show the noticeable changes that occur in the lattice dynamics and hence in the low-temperature properties of both glass and crystal states when chemical (position) isomers are introduced, namely by comparing 1-propanol vs 2-propanol and the different isomers of butanol.

\section{Experimental techniques}

The experiments discussed in the present work include calorimetric and specific-heat measurements, performed either at liquid nitrogen or at liquid helium temperatures and using different calorimetric methods, low-temperature thermal conductivity measurements in the range $2-120 \mathrm{~K}$, Brillouin-scattering experiments in the range 10-300 K, and $\mathrm{x}$-ray diffraction experiments between liquid-nitrogen and room temperatures.

As described in more detail elsewhere [4], we have implemented a quasiadiabatic continuous calorimetric system for glass-forming liquids that allows in situ preparation and calorimetric characterization of the different phases of the substance (typically using liquid nitrogen as thermal sink), followed by accurate heat-capacity measurements at low temperatures [5], where the liquid nitrogen bath is readily replaced by liquid helium, and using then two alternative thermal relaxation methods [4].

The thermal conductivity of the different samples was measured under equilibrium vapor pressure in an experimental set-up already described [6], using the steady-state potentiometric method.

High-resolution Brillouin-scattering measurements of both longitudinal and transverse sound velocities of the different samples in their glass state at low temperature were conducted by using an $\mathrm{Ar}^{+}$ion laser (wavelength = $=514.5 \mathrm{~nm}$ ) and a Sandercock-type 3+3 tandem FabryPérot interferometer [7]. In order to obtain direct information of the sound propagation velocities, the 90A scattering geometry was used.

The structure of the liquid and solid states of the different monoalcohols was investigated by means of x-ray diffraction experiments. An x-ray diffractometer D-500 (Siemens) with a secondary graphite monochromator and $\mathrm{Cu}-\mathrm{K}_{\alpha}$ ra- diation was used in transmission geometry. As described in more detail in Ref. 8, the liquid sample was inserted into a disk-like sample holder with beryllium disks of $\sim 20 \mathrm{~mm}$ in diameter as windows. The thickness of the beryllium disks is $\sim 0.1 \mathrm{~mm}$ and the distance between windows is $\sim 1.0 \mathrm{~mm}$.

\section{Experimental results}

\subsection{Methanol}

The first primary monoalcohol of the series $(n=1)$ is methanol or methyl alcohol, $\mathrm{CH}_{3} \mathrm{OH}$, which presents a small, "quasi-spherical" molecule and readily crystallizes. Hence it is very difficult to obtain pure methanol as bulk glass for standard conditions and cooling rates in typical calorimetric experiments [9]. The structure of both the liquid and the two low-temperature crystal phases found at ambient pressure consists of linear chains of alternating hydrogen bonded molecules [10]. Some researchers have studied methanol with a few molar percent of water as to avoid crystallization, but that is certainly another substance. Therefore, we have not measured the thermal properties of methanol.

\subsection{Ethanol}

Whereas pure methanol readily crystallizes and cannot be obtained in the glass state for standard conditions and cooling rates, ethanol exhibits a very rich polymorphism presenting four different solid states. Thus, ethanol or ethyl alcohol, $\mathrm{CH}_{3} \mathrm{CH}_{2} \mathrm{OH}$ (i.e., $n=2$ ), is not an excellent glassformer but at least the glass state can be readily obtained by supercooling the liquid at $30 \mathrm{~K} / \mathrm{min}$ [11], or even at slower cooling rates above $6 \mathrm{~K} / \mathrm{min}$ [12-15], since the critical cooling rate for ethanol has been found to depend on several factors such as minor water impurity, mechanical or geometrical details of the experimental cell driving heterogeneous crystallization and even on the previous thermal history of the liquid [16].

As said above, pure ethanol (see Fig. 1) exhibits a very rich polymorphism presenting four different solid states [11-13]: the ordinary amorphous solid or glass state obtained when the liquid is cooled sufficiently fast, a bodycentred cubic plastic crystal (PC) with rotational disorder which by quenching becomes an orientationally-disordered crystal (ODC), and a fully-ordered (monoclinic) crystal state with 4 atoms per unit cell [17], which in turn exists in four distinct phases [14], depending on temperature and thermal history. Therefore, a relatively simple and easily available chemical substance as ethanol offers an extremely useful benchmark to study the glass transition phenomenon and the very nature of glasses and amorphous solids, since one can choose and discriminate the roles played by translational, rotational and orientational disorder.

Very interestingly, both the standard glass transition (amorphous solid $\leftrightarrow$ supercooled liquid) and that dynami- 


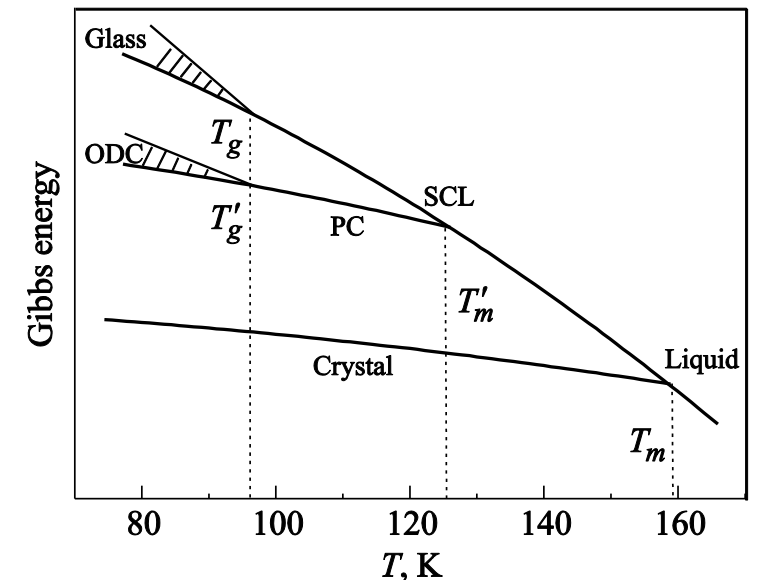

Fig. 1. Schematic Gibbs-energy phase diagram for ethanol. When the supercooled liquid (SCL) below the melting point $\left(T_{m}=159 \mathrm{~K}\right)$ is quenched fast enough, the glass state is obtained at $T_{g} \approx 97 \mathrm{~K}$. By heating the glass above $T_{g}$ or by cooling the SCL more slowly, a plastic crystal (PC) is obtained, which becomes an orientationally-disordered crystal (ODC) below a glasslike transition also at $T_{g}^{\prime} \approx 97 \mathrm{~K}$.

cal freezing of the PC into the ODC were found to occur at the same temperature $\left(T_{g} \approx T_{g}^{\prime} \approx 97 \mathrm{~K}\right)$ and with comparable discontinuities in the specific heat $[11,13,16]$, as shown in Fig. 2(a). Furthermore, the ODC state also exhibits, qualitatively and almost quantitatively, the same universal glassy properties at low temperatures such as tunneling states [5], the plateau in the thermal conductivity [18] or the boson peak $[13,15]$, as the truly amorphous, structural glass state, and in clear contrast to the stable (monoclinic) crystal (see Fig. 3(a)). For those reasons, such ODCs are sometimes also named "glassy crystals" or "orientational glasses".

\subsection{Propanol}

By putting a third carbon atom (i.e., adding another ethyl group) to the monoalcohol chain, one gets for $n=3$ the best glass-forming substance of the series: 1-propanol or $n$-propanol. This fact is evidenced by a narrower temperature range for the supercooled liquid than in ethanol. The glass transition temperatures are essentially the same, $T_{g} \approx 98 \mathrm{~K}$ for 1-propanol, but the melting point is now $11 \mathrm{~K}$ lower: $T_{m}=148 \mathrm{~K}$. Indeed complete crystallization of 1-propanol requires annealing over several hours at temperatures within a narrow range about $135 \mathrm{~K}[19,20]$. Its isomer 2-propanol is also a rather good glass-former, with higher transition temperatures, $T_{g} \approx 115 \mathrm{~K}$ and $T_{m}=185 \mathrm{~K}$, but a similar discontinuity of $\Delta C_{p}\left(T_{g}\right) \approx 45 \mathrm{~J} /(\mathrm{mol} \cdot \mathrm{K}$ ) (see the inset in Fig. 1 of Ref. 19). Surprisingly, 2-propanol was found [19] to exhibit a much higher boson peak (the broad maximum in $C_{p} / T^{3}$ ) than 1-propanol. This was attributed to a correspondingly higher density of Debye-like acoustic excitations, since the cubic Debye coefficients of the corresponding crystals presented a similar ratio (see Fig. 3(b)).
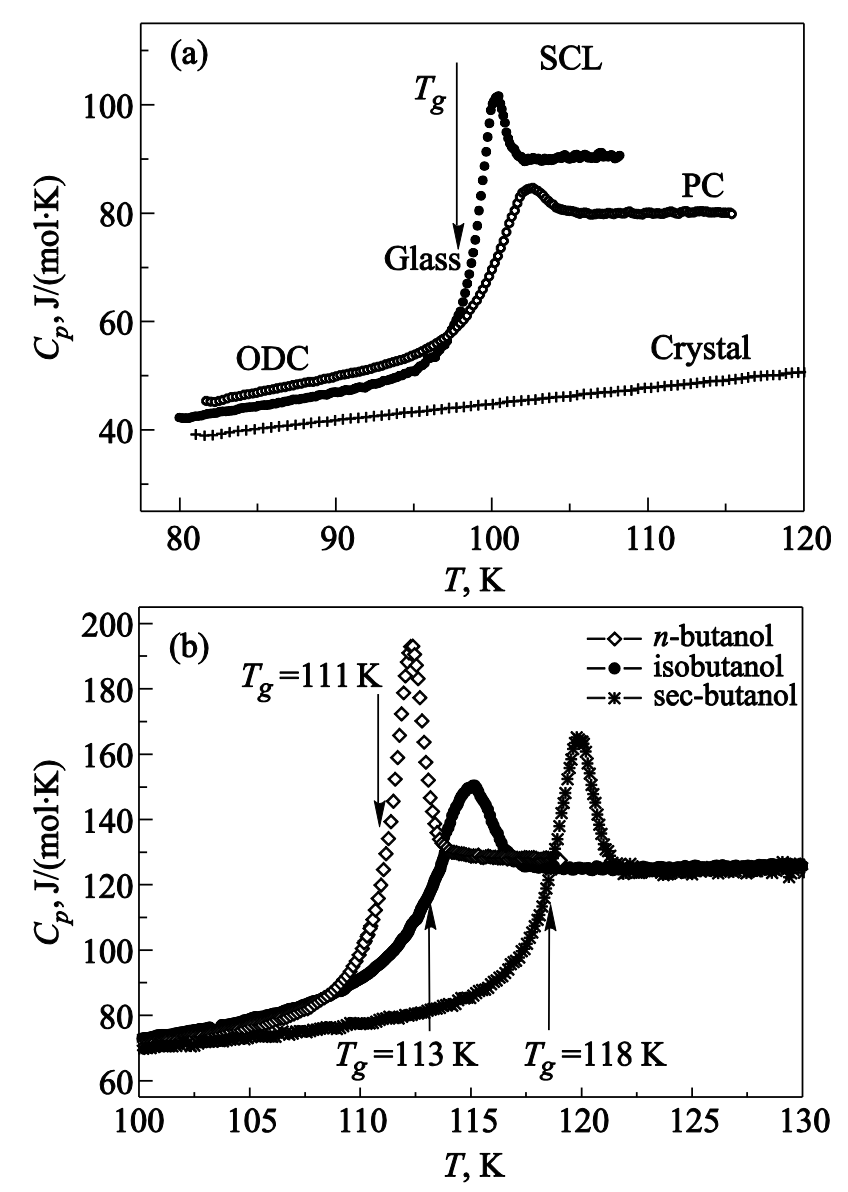

Fig. 2. Specific heat measured around the corresponding glass transitions for ethanol: solid circles show the glass-supercooled liquid (SCL) transition at $T_{g} \approx 97 \mathrm{~K}$, open circles show the analogous transition from the orientationally-disordered crystal (ODC) to the plastic crystal (PC), and crosses are data for the stable crystal (a); butanol: the symbols for the three position isomers with glass states are indicated in the legend, and the similar glass-transition temperatures are labelled in the plot (b).

\subsection{Butanol}

By further elongating the molecule $(n=4)$, the glassforming tendency begins to reverse: 1-butanol ( $n$-butanol) glass can be easily obtained when cooling the liquid at a moderately slow rate, but it crystallizes when heating the glass or if the supercooled liquid is kept at certain temperatures long enough. Interestingly, a metastable phase (between the glass and crystal ones) was found in 1-butanol [21], very similar to that previously found in triphenyl phosphite (TPP) $[22,23]$. In both cases, a new solid phase denoted as glacial phase or glacial state [22], obtained by a first-order, exothermic transformation from the supercooled liquid state was observed. Among other interpretations, this controversial "glacial phase" was firstly attributed in TPP to a defect-ordered phase within the theory of frustration-limited domains [22], and later was considered (both for TPP and 1-butanol) as a manifestation of liquidliquid transitions between two distinct liquid (with their corresponding amorphous solid) states [23]. A brief review 

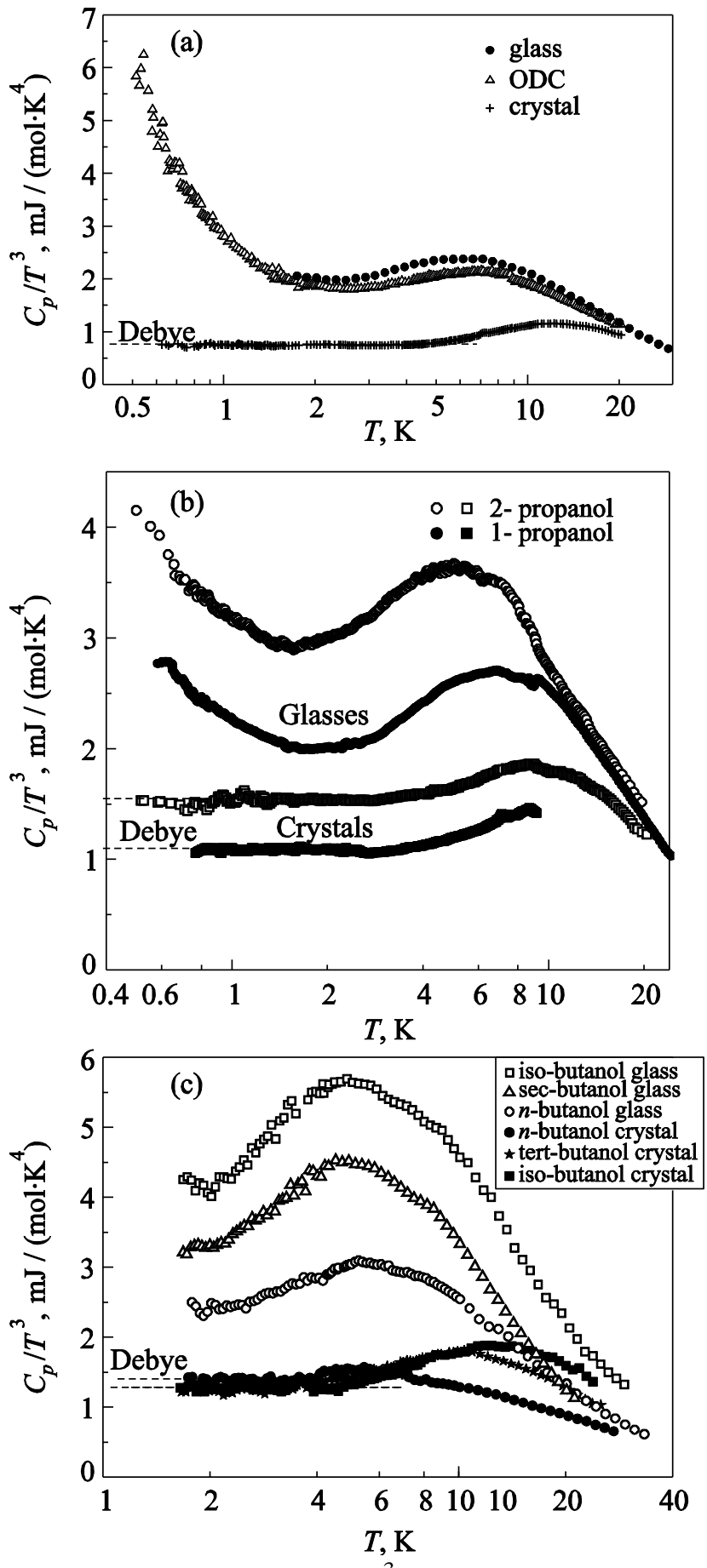

Fig. 3. Reduced specific-heat $C_{p} / T^{3}$ plots for glasses and crystalline phases of: pure ethanol, including the orientationallydisordered crystal (ODC) (a); 1-propanol and 2-propanol (b); different position isomers of butanol, as indicated in the legend. In all cases, the obtained Debye levels for the crystals are shown with dashed lines (c).

about polyamorphism with special emphasis on the case of TPP can be found in Ref. 3 .

Nonetheless, Hédoux et al. [24] have perfomed several experiments on TPP, and have shown that the atypical transformation kinetics found can be understood as a consequence of a time lag of nucleation, inducing a high nuc- leation rate which frustrates the crystallization and leads to the so-called "glacial state". By using Raman-scattering, the origin of the frustration of the crystallization in 1-butanol has also been explained [25] from the consideration that the rearrangement of the hydrogen-bonded molecules is unavoidably prevented by the very low molecular mobility of the system only a few degrees above $T_{g}$. The "glaciation" process would be an abortive crystallization driven by the formation of a layered, two-dimensional hydrogenbond network. This particular transformation kinetics can nonetheless be described [24,25] by a classical nucleationgrowth law. In fact, the temporal evolution of the transformation degree in 1-butanol at two different temperatures above $T_{g}$ were found [25] to follow a clear Avrami sigmoidal shape, indicative of a first-order character for the isothermal transformation. The authors estimated degrees of crystallinity at the end of the transformation processes typically around 70-80\% [25]. Our recent experiments $[7,8,26]$ fully support this view that the "glacial phase" of 1-butanol is just a mixture of many nanocrystallites embedded in a disordered matrix, presumably due to an aborted crystallization originated by a high nucleation rate in a temperature range where the crystal growth is low. In addition, the stable crystalline state was found to present triclinic symmetry with 6 atoms per unit cell [8].

Finally, we have concurrently studied [27] the lowtemperature thermal properties of the different position isomers of butanol ( $n-$, sec-, tert- and iso-butanol), though only in three of them we were able to obtain the glass state: in tertbutanol only crystalline phases were observed. In Fig. 2(b) we show the obtained specific-heat data around the glass transition for $n$-butanol (= 1-butanol), sec-butanol (=2-butanol) and iso-butanol. Also, in three out of the four cases, we were able to obtain the fully crystalline state and measured their specific heat as a useful reference. Brillouin-scattering measurements at low temperatures of longitudinal and transverse sound velocities for the different glasses provided us with the Debye phonon-like contribution to their specific-heat curves. Complementary thermalconductivity measurements for those butanol glasses, performed between $2 \mathrm{~K}$ and the glass transition temperatures allowed us to carry out an exhaustive study [27] for different glasses of the same substance, i.e., butanol, in order to assess the effects of changing the spatial arrangement of atoms and the position of hydrogen bonds in the lattice on the low-temperature thermal and elastoacoustic properties around the universal boson peak feature in glasses. In brief, we found that the main thermal properties of butanol glasses at low temperatures strongly vary among its different position isomers, when the hydrogen bond of the hydroxyl group $(\mathrm{OH})$ locates in a different position of the butanol molecule, in clear contrast with the elastic Debye contributions, since both the Debye specific heat of the reference crystals and that of glasses obtained from Brillouin experiments remain essentially constant for the dif- 
ferent isomers (see Fig. 3(c)). Moreover, we want to stress that a consistent analysis of all low-temperature thermal properties of butanol glasses using the soft-potential model [28] was possible [27].

\section{Discussion}

We will firstly focus on the primary monoalcohols. As shown above, by gradually increasing the "length" of the molecule, one modifies the balance between crystallization kinetics and glass-forming ability. Whereas pure methanol (a small, "quasi-spherical" molecule) readily crystallizes and we were not able to obtain it in the glass state for standard conditions and cooling rates, ethanol seems to possess a "critical" aspect ratio for its molecule, with competing interactions and driving forces which are likely at the origin of its interesting and rich polymorphism, depicted in Fig. 1. By adding another ethyl group to the hydrocarbon backbone and hence further elongating the "ellipsoidal" molecule, the best glass-forming primary monoalcohol is observed: 1-propanol. Propanol molecules seem to be long enough as to seriously hinder the crystallization arrangement. With the next in the series, the glass-forming tendency begins to reverse: 1-butanol glass can be easily obtained when cooling the liquid at a moderately slow rate, but it readily crystallizes when heating the glass, though often in a two-step process that could be wrongly taken as a polyamorphic transition. Interestingly, this glass-forming tendency found in primary monohydroxy alcohols is exactly the same previously observed for the first four members of the alkyl-cyclohexanes family [29]. It suggests therefore that when the hydrocarbon molecules are long enough, they begin to find easier to align themselves and crystallize, and this crossover typically occurs when passing from 3 to 4 carbon atoms in the linear chain.

In Table 1, we summarize the main calorimetric and thermodynamic data of all studied monoalcohols. We include the value of the specific-heat discontinuity at the glass transition in units of the gas constant, $\Delta C_{p}\left(T_{g}\right) / R$, as a measure of the number of degrees of freedom that are released at the glass transition. It is noteworthy that $\Delta C_{p}$ $\left(T_{g}\right) / R$ does not increase more or less linearly with the size of the molecule (i.e., with $n$ ), but it remains rather constant. Also, the position and height of the boson peak in the different glasses (including the "glassy crystal" of ethanol, ODC) is compared to the Debye contribution found in the corresponding crystals. No clear correlation between them is found. In this case, however, the amount of lowtemperature glassy excitations per mol roughly increases with the size of the molecule.

\section{Conclusions}

We have discussed how either the gradual elongation of the monoalcohol molecule with increasing number of ethyl groups or the hydrogen-bonding location have a strong influence on the glass-forming ability, as well as on lowfrequency dynamics and low-temperature properties of molecular solids. The competition between crystallization rate and glass-forming ability seems to depend mainly on the aspect ratio of the molecules, ranging from the easiest crystal former methanol to the best glass former propanol. Intermediate glass formers as ethanol or butanol has been argued to present the added value of a rich polymorphism -the former-and the possibility of comparing different chemical isomers -the latter-. However, the claimed polyamorphism reported to exist in $n$-butanol ascribed to a new glacial state was found to be just a mixture of nanocrystallites embedded in a glassy matrix, presumably due to an aborted crystallization originated by a high nucleation rate in a temperature range where the crystal growth is low.

On the other hand, it was found that the low-energy glassy excitations (tunneling states, boson peak...) of the structural (amorphous) and orientational (ODC) glasses of ethanol were very similar, whereas those of secondary alcohols were unexpectedly larger than those of their corresponding primary alcohols. Nonetheless, in the case of pro-

Table 1. Calorimetric and thermodynamic data of several monohydroxy alcohols. $T_{g}$ is the glass-transition temperature and $\Delta C_{p}\left(T_{g}\right)$ its specific-heat discontinuity; $R$ is the gas constant; $T_{m}$ is the melting temperature; $C_{D}$, crystal is the Debye cubic coefficient obtained from the crystal specific heat; $T_{\max , B P}$ is the temperature of the maximum in $C_{p} / T^{3}$ (boson peak) and $\left(C_{p} / T^{3}\right)_{B P}$ its height.

\begin{tabular}{|c|c|c|c|c|c|c|c|c|}
\hline Substance & $\begin{array}{c}T_{g}, \\
\mathrm{~K}\end{array}$ & $\begin{array}{c}\Delta C_{p}\left(T_{g}\right), \\
\mathrm{J} \cdot \mathrm{mol}^{-1} \cdot \mathrm{K}^{-1}\end{array}$ & $\Delta C_{p} / R$ & $\begin{array}{c}T_{m}, \\
\mathrm{~K}\end{array}$ & $\begin{array}{c}C_{D, \text { crystal }}, \\
\mathrm{mJ} \cdot \mathrm{mol}^{-1} \cdot \mathrm{K}^{-4}\end{array}$ & $T_{\max , B P}$, & $\begin{array}{c}\left(C_{p} / T^{3}\right)_{B P}, \\
\mathrm{~mJ} \cdot \mathrm{mol}^{-1} \cdot \mathrm{K}^{-4}\end{array}$ & Refs. \\
\hline methanol & 103 & 30 & 3.6 & 176 & - & - & - & 9 \\
\hline ethanol (glass) & 97 & 35.3 & 4.2 & 159 & 0.766 & 6.1 & 2.4 & 15,16 \\
\hline ethanol (ODC) & 97 & 22.8 & 2.7 & 127.5 & 0.766 & 6.8 & 2.2 & 15,16 \\
\hline 1-propanol & 98 & 45 & 5.4 & 148 & 1.10 & 6.7 & 2.7 & 15,19 \\
\hline 2-propanol & 115 & 46 & 5.5 & 185 & 1.55 & 5.0 & 3.6 & 15,19 \\
\hline (1-) $n$-butanol & 111 & 48 & 5.8 & 184 & 1.40 & 5.4 & 3.1 & 8,27 \\
\hline (2-) sec-butanol & 118 & 40 & 4.8 & 185 & - & 4.8 & 4.6 & 27 \\
\hline tert-butanol & - & - & - & 293 & 1.28 & - & & 27 \\
\hline isobutanol & 113 & 40 & 4.8 & 168 & 1.28 & 4.8 & 5.7 & 27 \\
\hline
\end{tabular}


panol, 2-propanol crystal exhibits a correspondingly larger Debye coefficient than 1-propanol crystal. In contrast, 2-butanol glass also exhibits a significantly higher low-temperature specific heat than that of 1-butanol, but the Debye specific heats of all butanol crystals are practically identical. Therefore, no universal correlation between the boson peak of glasses and the Debye-like elastic properties can be drawn.

\section{Acknowledgments}

The Laboratorio de Bajas Temperaturas (UAM) is an associated unit with the ICMM-CSIC. This work was partially supported by the Spanish MINECO (FIS2011-23488 and Consolider Ingenio Molecular Nanoscience CSD200700010 program) and by the Comunidad de Madrid through program Nanobiomagnet (S2009/MAT-1726).

1. See, for instance, P.W. Anderson, Science 267, 1615 (1995).

2. Amorphous Solids: Low Temperature Properties, W.A. Phillips (ed.), Springer-Verlag, Berlin, Heidelberg (1981).

3. J. Senker and E. Rössler, Chem. Geol. 174, 143 (2001).

4. E. Pérez-Enciso and M.A. Ramos, Termochim. Acta 461, 50 (2007).

5. C. Talón, M.A. Ramos, and S. Vieira, Phys. Rev. B 66, 012201 (2002).

6. A.I. Krivchikov, V.G. Manzhelii, O.A. Korolyuk, B.Ya. Gorodilov, and O.O. Romantsova, Phys. Chem. Chem. Phys. 7, 728 (2005).

7. M. Hassaine, R.J. Jiménez-Riobóo, I.V. Sharapova, O.A. Korolyuk, A.I. Krivchikov, and M.A. Ramos, J. Chem. Phys. 131, 174508 (2009).

8. I.M. Shmyt'ko, R.J. Jiménez-Riobóo, M. Hassaine, and M.A. Ramos, J. Phys.: Condens. Matter 22, 195102 (2010).

9. M. Sugisaki, H. Suga, and S. Seki, Bull. Chem. Soc. Jpn. 41, 2586 (1968).

10. 10. K.J. Tauer and W.N. Lipscomb, Acta Crystallogr. 5, 606 (1952); B.H. Torrie, S.X. Weng, and B.M. Powell, Mol. Phys. 67, 575 (1989).

11. O. Haida, H. Suga, and S. Seki, J. Chem. Thermodyn. 9, 1133 (1977).

12. A. Srinivasan, F.J. Bermejo, A. de Andrés, J. Dawidowski, J. Zúñiga, and A. Criado, Phys. Rev. B 53, 8172 (1996).
13. M.A. Ramos, S. Vieira, F.J. Bermejo, J. Dawidowski, H.E. Fisher, H. Schober, M.A. González, C.K. Loong and D.L. Price, Phys. Rev. Lett. 78, 82 (1997).

14. M.A. Ramos, I.M. Shmyt'ko, E.A. Arnautova, R.J. JiménezRiobóo, V. Rodríguez-Mora, S. Vieira, and M.J. Capitán, J. Non-Cryst. Solids 352, 4769 (2006).

15. M.A. Ramos, C. Talón, R.J. Jiménez-Riobóo, and S. Vieira, J. Phys.: Condens. Matter 15, S1007 (2003).

16. B. Kabtoul, R.J. Jiménez-Riobóo, and M.A. Ramos, Philos. Mag. 88, 4197 (2008).

17. P.G. Jönsson, Acta Crystallogr. Sect. B 32, 232 (1976).

18. A.I. Krivchikov, A.N. Yushchenko, V.G. Manzhelii, O.A. Korolyuk, F.J. Bermejo, R. Fernández-Perea, C. Cabrillo, and M.A. González, Phys. Rev. B 74, 060201 (2006).

19. C. Talón, M.A. Ramos, S. Vieira, I. Shmyt'ko, N. Afonikova, A. Criado, G. Madariaga, and F.J. Bermejo, J. Non-Cryst. Solids 287, 226 (2001).

20. C. Talón, F.J. Bermejo, C. Cabrillo, G.J. Cuello, M.A. González, J.W. Richardson, Jr., A. Criado, M.A. Ramos, S. Vieira, F.L. Cumbrera, and L.M. González, Phys. Rev. Lett. 88, 115506 (2002).

21. B.V. Bol'shakov and A.G. Dzhonson, Dokl. Phys. Chem. 393, 318 (2003); B.V. Bol'shakov and A.G. Dzhonson, J. Non-Cryst. Solids 351, 444 (2005).

22. A. Ha, I. Cohen, X. Zhao, M. Lee, and D. Kivelson, J. Phys. Chem. 100, 1 (1996).

23. H. Tanaka, R. Kurita, and H. Mataki, Phys. Rev. Lett. 92, 025701 (2004); R. Kurita, and H. Tanaka, Science 306, 845 (2004); R. Kurita and H. Tanaka, J. Phys.: Condens. Matter 17, L293 (2005).

24. A. Hédoux, Y. Guinet, M. Foulon, and M. Descamps, $J$. Chem. Phys. 116, 9374 (2002).

25. A. Wypych, Y. Guinet, and A. Hédoux, Phys. Rev. B 76, 144202 (2007).

26. A.I. Krivchikov, M. Hassaine, I.V. Sharapova, O.A. Korolyuk, R.J. Jiménez-Riobóo, and M.A. Ramos, J. Non-Cryst. Solids 357, 524 (2011).

27. M. Hassaine, M.A. Ramos, A.I. Krivchikov, I.V. Sharapova, O.A. Korolyuk, and R.J. Jiménez-Riobóo, Phys. Rev. B 85, 104206 (2012).

28. M.A. Ramos and U. Buchenau, Phys. Rev. B 55, 5749 (1997).

29. A. Mandanici, M. Cutroni, A. Triolo, V. Rodriguez-Mora, and M.A. Ramos, J. Chem. Phys. 125, 054514 (2006). 